\title{
Water Absorption and Degradation Characteristics of Chitosan-Based Polyesters and Hydroxyapatite Composites
}

\author{
Vitor M. Correlo, Elisabete D. Pinho, Iva Pashkuleva, Mrinal Bhattacharya,* \\ Nuno M. Neves, Rui L. Reis
}

Blends of chitosan and biodegradable synthetic aliphatic polyesters (polycaprolactone, poly(butylene succinate), poly[(butylene succinate)-co-adipate], poly[(butylene terephthalate)co-adipate], and poly(lactic acid)) were injection-molded. These samples were immersed in isotonic solution at $37^{\circ} \mathrm{C}$ for a period of $60 \mathrm{~d}$. The water uptake and the degradation properties, as measured by the loss in tensile strength, were evaluated as a function of time. In this study, the rate and the equilibrium water uptake were proportional to the amount of chitosan in the blend. The addition of HA to chitosan and polyester significantly reduced the equilibrium water uptake. The water uptake did not follow the classical Fickian phenomena and could be expressed by a two-stage sorption non-Fickian diffusion model. Contact angle measurement was used to quantify the changes in surface hydrophilicity as a function of chitosan and polyester composition. The glycerol contact angle decreased with increasing synthetic components in the blend. The blends and composites also showed increased degradation, as quantified by a loss in their mechanical properties, with increase in natural content. The degradation of properties was directly related to the water uptake of the blends; the higher the water uptake, the higher the degradation. Pure polyesters, while having low water uptake, nevertheless showed significant degradation by a precipitous drop in the strain at break. Among the polyesters, poly(lactic acid) displayed maximum degradation, while polycaprolactone displayed the least.

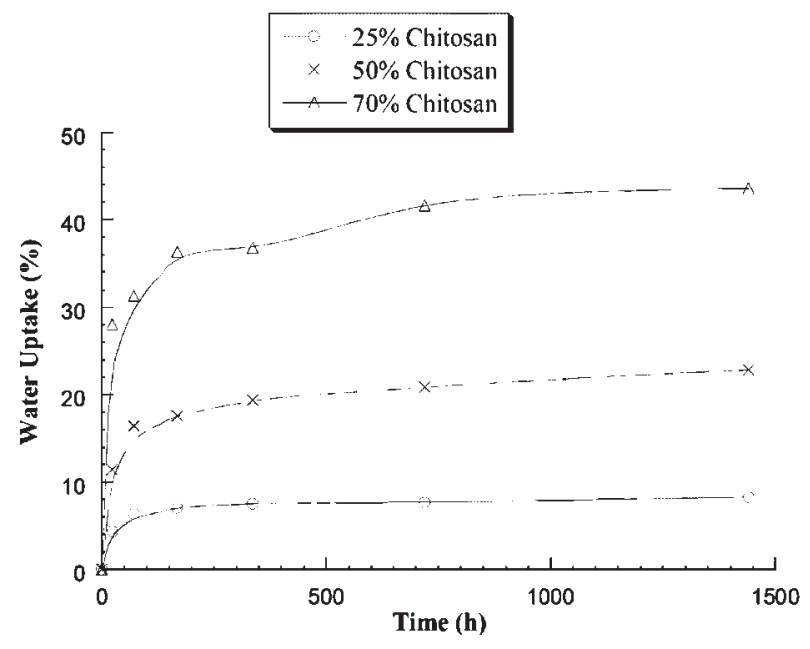

V. M. Correlo, E. D. Pinho, I. Pashkuleva, M. Bhattacharya, N. M. Neves, R. L. Reis 3B's Research Group, Department of Polymer Engineering, University of Minho, Guimarães, Portugal

E-mail: bhattoo2@umn.edu

M. Bhattacharya

Current Address: M. Bhattacharya, Department of Bioproducts and Biosystems Engineering, University of Minnesota, St. Paul,

Minnesota 55108, USA

\section{Introduction}

Water uptake of polymeric implants affects their mechanical properties, degradability, and dimensional stability. These defects have the potential of compromising function and biocompatibility. Water exposure and uptake may decrease the life of an implant due to hydrolysis and 
microcrack formation. The amount of water uptake is determined by the diffusion coefficient of the material. High diffusion coefficients allow water to penetrate into the matrix enabling water-soluble additives, including growth factors that aid in tissue repair, to be released more rapidly. Studies have also shown that equilibrium water content and the organization of water within the matrix affect cell adhesion. ${ }^{[1]}$

Similarly, the degradation of the polymeric matrix is of importance in the production and use of implantable devices, particularly those involved in bone fixation, bone regeneration, and tissue engineering. ${ }^{[2,3]}$ The rate of degradation of these implants (loss in mechanical properties) should be tailored to the rate of tissue generation. The control of the hydrophilicity of a material helps in controlling the degradation rate of the material. ${ }^{[4]}$

Another important property of a biomaterial is its surface characteristics. There has been major interest in these characteristics since it is the surface of these materials that first comes into contact with the biological surroundings. The change in the surface properties was found to affect the interaction of the surface with biomacromolecules, such as proteins, ${ }^{[5-7]}$ and with cells. ${ }^{[7-9]}$ The energy of the surface, which is directly related to its wettability, is a useful parameter that has often correlated strongly with those biological interactions.

We have recently reported on the properties and morphology of chitosan-based polyester blends. ${ }^{[10,11]}$ This work aims at reporting on the water absorption and degradation of the newly developed polymer blends and composites since these properties are critical for their possible applications as biomaterials.

\section{Experimental Part}

\section{Materials}

The chitosan/polyester blends were compounded in a twin-screw extruder. The polyesters used include poly( $\varepsilon$-caprolactone) (PCL), poly(butylene succinate) (PBS), poly(lactic acid) (PLA), poly(butylene terephthalate adipate) (PBTA), and poly(butylene succinate adipate) (PBSA). The chitosan used had a degree of deacetylation of approximately $85 \%$. Briefly, the chitosan/polyester blends were prepared by melt blending in a twin-screw extruder. These blends were then further compounded with hydroxyapatite (HA). In this study, the formulations of chitosan/polyester will be referred to as blends and the formulations containing chitosan/polyester/HA as composites. The details of the processing conditions are summarized elsewhere. ${ }^{[10,11]}$ The extruded strands were ground to $5 \mathrm{~mm}$ diameter pellets using a Coloritron grinder. The blends were injection-molded using an ENGEL injection molding machine to produce tensile test bars. The tensile bars had a neck cross-section area of $2 \times 4 \mathrm{~mm}^{2}$ and a neck length of $20 \mathrm{~mm}$.

\section{Water Absorption}

The molded samples were dried in a vacuum oven at $50^{\circ} \mathrm{C}$ until a constant weight was obtained. These samples were immersed in an isotonic solution of $\mathrm{NaCl} 0.154 \mathrm{M}\left(9 \mathrm{~g} \cdot \mathrm{l}^{-1}\right)$ and $\mathrm{pH} 7.4$ at $37^{\circ} \mathrm{C}$ for periods of $1,3,7,14,30$, and $60 \mathrm{~d}$. The samples were then removed at specific intervals, gently blotted with tissue paper to remove the excess water on the surface, and the weight recorded. This process was repeated at several time intervals. In order to ensure that no leaching had occurred, samples were dried at the end of the test period and weighed and compared against the original sample weight. Where leaching was found to occur, the data were corrected to account for the weight loss. The parameters $D, \phi$, and $\psi$ were estimated for the data by non-linear regression routine (provided in Kaleidagraph, Synergy Software, Reading, PA, USA) based on a modified Levenberg-Marquardt algorithm.

\section{Degradation}

The injection-molded samples were immersed in an isotonic saline solution for periods of 1, 3, 7, 14, 30, and $60 \mathrm{~d}$. A solution of $40 \mathrm{ml}$ was used for three samples (one batch). Two batches were used for each selected immersion period. At the end of each immersion period, the solution $\mathrm{pH}$ was measured. The weight loss was determined by drying the samples to constant weight and comparing to their initial weight. The tensile bars from the water absorption tests above were subjected to tensile testing using an Instron Universal Tensile Testing Machine. The tensile modulus was the initial slope of the force-deformation curve.

\section{Contact Angle}

The static contact angle measurements were obtained by the sessile drop method using a contact angle meter OCA15+ with a high-performance image processing system (DataPhysics Instruments, Germany). The liquid (glycerol or $\mathrm{CH}_{2} \mathrm{I}_{2}, 1 \mu \mathrm{l}$, HPLC grade) was added by a motor-driven syringe at room temperature. Five samples of each material were used, and six measurements were carried out for each sample. The data presented are an average of five readings. The polarity of the surface and the surface tension were calculated using Kaelble's equation.

\section{Results and Discussions}

\section{Water Absorption}

The fractional water-uptake curves as a function of chitosan content and type of polyesters are shown in Figure 1 and 2. As the chitosan content was increased, the water uptake increased and the time required to achieve the equilibrium water content increased. This was an expected result since chitosan is hydrophilic and the aliphatic polyesters are hydrophobic. Similar results are reported in the literature for polyester blends with other natural origin polymers like starch. ${ }^{[12,13]}$ This is primarily due to the presence of amine and hydroxyl groups on the chitosan moiety, which is the most probable site for 


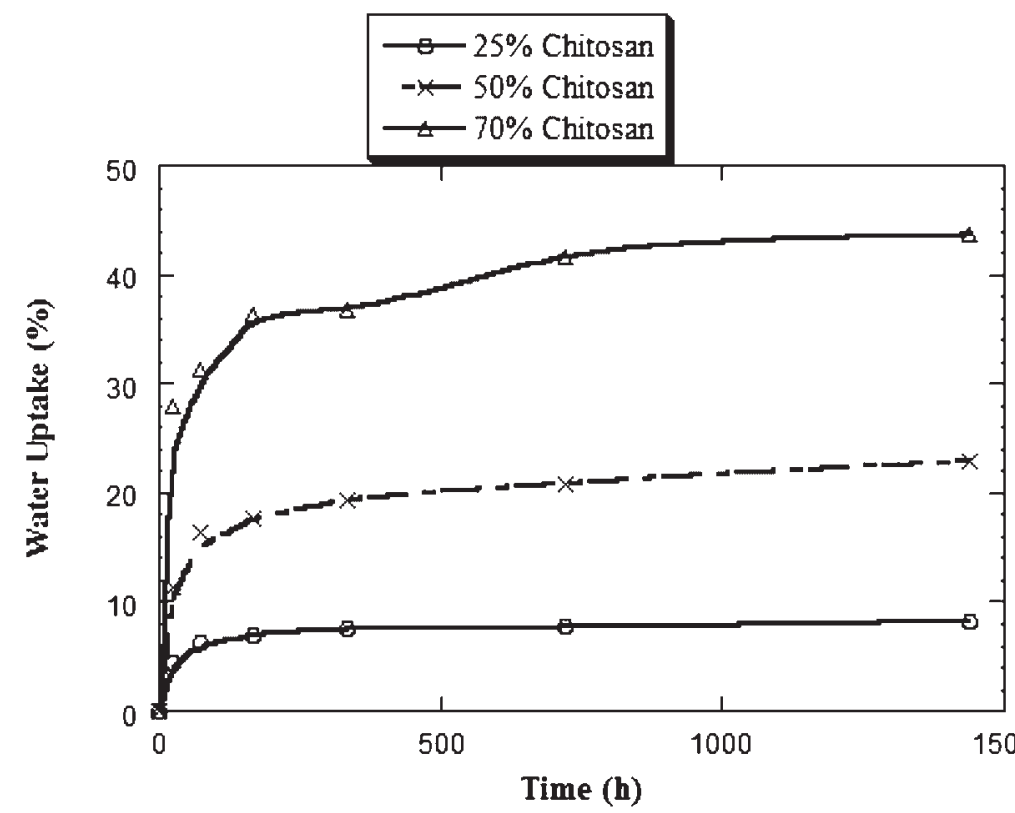

Figure 1. Water uptake as a function of chitosan content for PBS/chitosan blends.

accommodation of the additional water. The water uptake characteristics of pure polyester (figure not shown) are typical of hydrophobic polymers, with PCL exhibiting the lowest equilibrium water uptake $(0.5 \%)$ and other polymers at approximately $1.5 \%$ at the end of $60 \mathrm{~d}$ of immersion.

The water absorption curves for blends containing $50 \%$ chitosan and different polyesters are shown in Figure 2. There is a sharp burst of water intake initially in all of the blends. Of the polyesters tested, the water uptake curves of

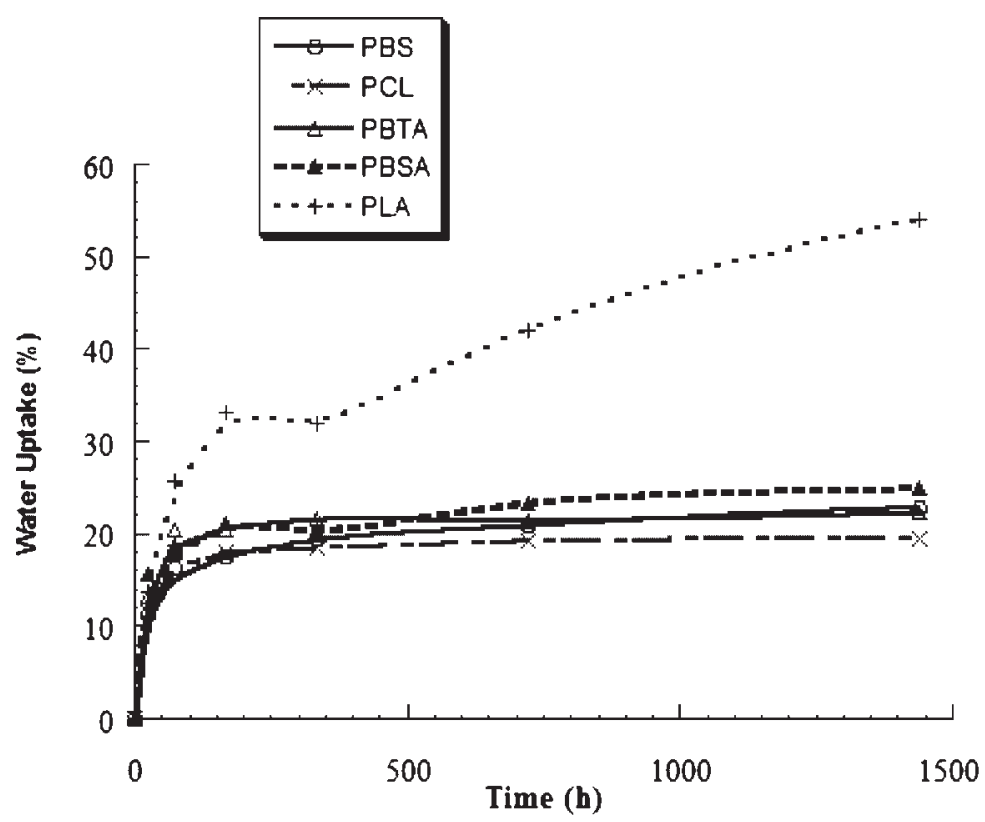

Figure 2. Water uptake for different polyester blends containing $50 \%$ chitosan.
PBS, PBSA, PCL, and PBTA are similar and have comparable times required to achieve equilibrium water uptake. The lowest equilibrium water content occurred with blends containing PCL. The highest water uptake was experienced with blends containing PLA. It was observed that the water absorption of PLA/chitosan blends does not attain an equilibrium value but displays a two-step kinetics. The first step is similar to other blends where the penetrant is picked up by the hydrophillic chitosan. SEM has shown that materials display a skin-core morphology with the skin being polyester rich while the core is a blend of chitosan and polyester. $^{[10]}$ Since chitosan is hydrophilic, it picks up water that diffuses into the polyester matrix even in the inner regions, increasing the equilibrium water uptake. The second step of the chitosan-PLA water uptake may be explained by the formation of micro-cracks in the surface (visible to the naked eye) of the specimens during the longer degradation stages. The higher water uptake could be the low crystallinity of PLA with respect to PBS, PCL, and PBSA. For PBTA, which is also mostly amorphous, the water absorption is sharply lower and thus can be attributed to the non-formation of micro-cracks. In an elastomerlike material the formation of micro-cracks is highly unlikely.

The addition of HA significantly reduces the equilibrium water uptake (Figure 3). Similar results were reported by Santos for BisGMA incorporated with HA. ${ }^{[14]}$ This is due to the decrease in the amount of the hydrophilic component (chitosan) in the composite. However, these composites containing HA showed a significantly sharper uptake of water in the first few hours and achieved equilibrium water uptake much quicker than that of chitosanbased blends (Figure 1 and 2 versus Figure 3). This can be explained from previous morphological observations using SEM that have shown that HA crystals have minimal adhesion to the polyester matrix. ${ }^{[11]}$ This is seen in Figure 4 where cavities with smooth surface were formed due to the extensive detaching of HA crystals. This increase can be attributed to the presence of spaces between the HA and the matrix, where the adsorbed water is lodged. The interfaces between the HA crystals and chitosan/polyester matrix will work as micro-voids that will facilitate water diffusion into the specimens and reach chitosan domains easier. This will allow faster water diffusion and consequently a quicker achievement of the equilibrium water uptake or saturation. 


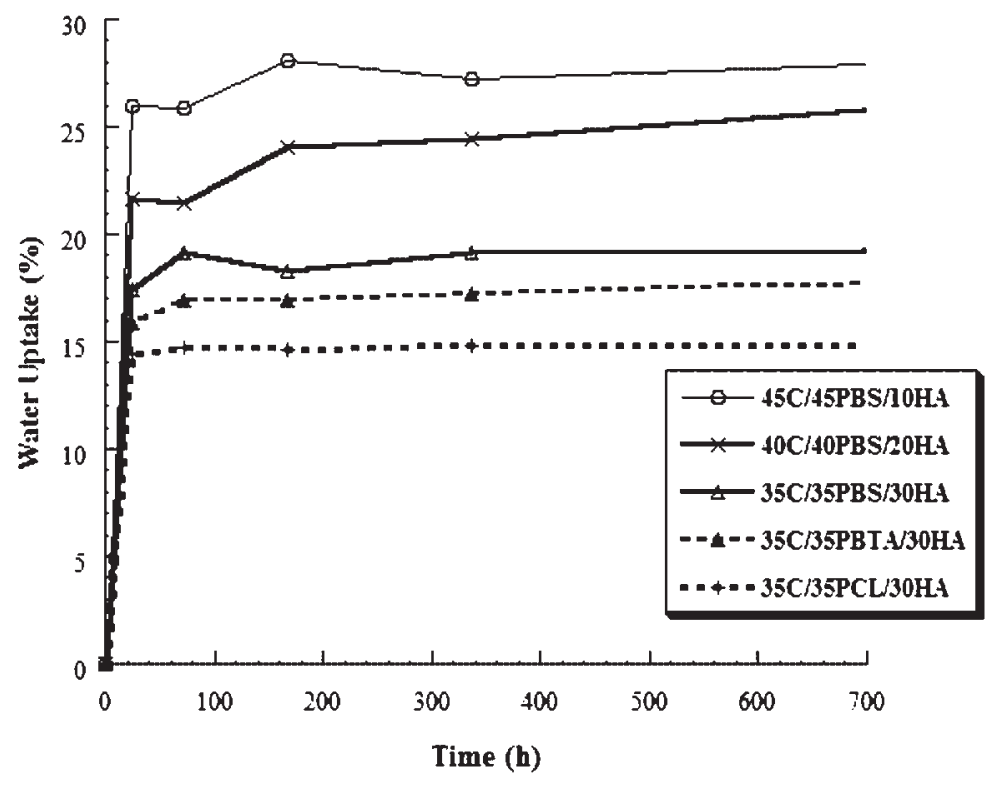

Figure 3. Water uptake for polyester/chitosan composites containing HA. where $M(t)$ is the water uptake at time $t, M_{\mathrm{eq}}$ the water uptake at $t=\infty$, and $L$ the thickness of the sample. Adherence to Fickian behavior is determined by testing the conformity to the initial kinetics to $t^{0.5}$ scaling. At small times, when $M(t) / M_{\text {eq }}$ is small (<0.60), Equation (2) can be approximated by

$$
\frac{M(t)}{M_{\mathrm{eq}}}=\frac{4}{L} \sqrt{\frac{D t}{\pi}}
$$

Plots of $M(t) / M_{\text {eq }}$ against $t^{0.5}$ were found to be non-linear in the initial stages indicating that the process of solvent uptake follows an anomalous process. When a solvent penetrates a polymer, the movement of polymer chains is not sufficiently rapid to completely homogenize the penetrant's environment. This is particularly true of blends of hydrophilic and hydrophobic polymers where the penetrants have

The diffusion of a solvent into a polymeric matrix has been modeled by several researchers using Fick's second law, expressed as ${ }^{[14-19]}$

$$
\frac{\partial C}{\partial t}=D \frac{\partial^{2} C}{\partial x^{2}}
$$

where $C$ denotes the concentration of the diffusing solvent at time $t$ along the axis $x$. If the initial concentration of the solvent is zero and the polymer is placed in an infinite bath of the solvent, the solution to Equation (1) is given by ${ }^{[20]}$

$$
\frac{M(t)}{M_{\mathrm{eq}}}=1-\frac{8}{\pi^{2}} \sum_{m=0}^{\infty} \frac{1}{(2 m+1)^{2}} \exp \left[-\frac{\pi^{2}(2 m+1)^{2}}{L^{2}} D t\right]
$$

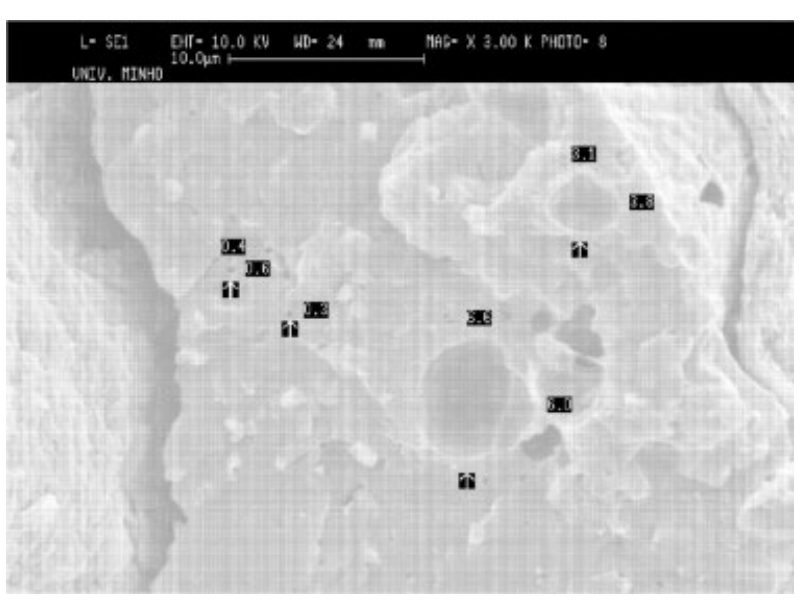

Figure 4. SEM image of the tensile fractured surface of the blend $35 \%$ chitosan, $35 \% \mathrm{PBS}$, and $30 \% \mathrm{HA}$. different diffusional mobilities. The relatively similar magnitude of the rates of diffusion and relaxation process leads to anomalous behavior during solvent uptake. On the other hand, when one dominates the other, Fickian diffusion is observed.

Morphological studies have shown that the blend systems are a two-phase system. ${ }^{[10]}$ Hence, one would expect that the water diffusion into the polyester phase is different from that in the chitosan phase and would result in some deviations from the typical Fickian type of water diffusion which was derived for more homogeneous matrices.

Two-stage sorption, a notable non-Fickian phenomenon, has been observed by several authors. ${ }^{[21,22]}$ Berens and Hopfenberg ${ }^{[21]}$ considered the sorption process as a linear superposition of phenomenologically independent contributions from Fickian diffusion and polymeric relaxation. The diffusion-controlled initial sorption is faster than the relaxation process, thus permitting explicit separation of the sorption process into two independent mechanisms. The resulting modification to Equation (2), assuming a first order relaxation process, results in the expression below:

$$
\begin{aligned}
\frac{M_{\mathrm{t}}}{M_{\infty}}= & \phi\left(1-\frac{8}{\pi^{2}} \sum_{m=0} \frac{1}{(2 m+1)^{2}} \exp \left(-\frac{(2 m+1)^{2} \pi^{2} D t}{L^{2}}\right)\right) \\
& +(1-\phi)\left[1-\exp \left(-\psi \frac{D t}{L^{2}}\right)\right]
\end{aligned}
$$

where $\phi$ is a measure of the ratio of the equilibria of the first stage to that of the second stage in the sorption, and $\psi$ is the ratio of the characteristic diffusion time $L^{2} / D$ to the characteristic time of relaxation.

The diffusion coefficients calculated using Equation (4) are summarized in Table 1 . The datasets for pure polymers were not considered since the differences between successive data points were too small to be determined 
Table 1. Water-uptake parameters for various blend and composites compositions.

\begin{tabular}{|c|c|c|c|c|c|}
\hline \multirow[t]{2}{*}{ Material composition } & \multirow{2}{*}{$\begin{array}{c}\text { Equilibrium water content } \\
\%\end{array}$} & \multirow{2}{*}{$\frac{D \times 10^{8}}{\mathrm{~m}^{2} \cdot \mathrm{h}^{-1}}$} & \multirow[t]{2}{*}{$\phi$} & \multirow[t]{2}{*}{$\psi$} & \multirow[t]{2}{*}{$R^{2}$} \\
\hline & & & & & \\
\hline $25 \mathrm{C} / 75 \mathrm{PBS}$ & 7.7 & 1.30 & 0.79 & 0.63 & 0.99 \\
\hline $50 \mathrm{C} / 50 \mathrm{PBS}$ & 21.4 & 1.61 & 0.68 & 0.52 & 0.99 \\
\hline $50 \mathrm{C} / 50 \mathrm{PCL}$ & 18.9 & 2.20 & 0.84 & 0.38 & 0.99 \\
\hline 50 C/50 PBTA & 22.4 & 1.30 & 0.92 & 0.48 & 0.99 \\
\hline $50 \mathrm{C} / 50$ PBSA & 23.9 & 2.56 & 0.73 & 0.30 & 0.99 \\
\hline 50 C/50 PLA & & 0.82 & 0.49 & 0.75 & 0.99 \\
\hline 70 C/30 PBS & 44.5 & 4.00 & 0.68 & 0.35 & 0.99 \\
\hline $17.5 \mathrm{C} / 52.5 \mathrm{PBS} / 30 \mathrm{HA}$ & 3.9 & 9.43 & 0.0006 & 4.16 & 0.99 \\
\hline $70 \mathrm{PBS} / 30 \mathrm{HA}$ & 1.8 & 11.30 & 0.72 & 0.09 & 0.99 \\
\hline $45 \mathrm{C} / 45 \mathrm{PBS} / 10 \mathrm{HA}$ & 24.2 & 17.62 & 0.88 & 0.24 & 0.99 \\
\hline $40 \mathrm{C} / 40 \mathrm{PBS} / 20 \mathrm{HA}$ & 19.1 & 15.46 & 0.80 & 0.12 & 0.99 \\
\hline $35 \mathrm{C} / 35 \mathrm{PBS} / 30 \mathrm{HA}$ & 8.3 & 4.02 & 0.95 & 0.39 & 0.99 \\
\hline $35 \mathrm{C} / 35 \mathrm{PBTA} / 30 \mathrm{HA}$ & 11.0 & 5.21 & 0.91 & 0.36 & 0.99 \\
\hline $35 \mathrm{C} / 35 \mathrm{PCL} / 30 \mathrm{HA}$ & 7.5 & 79.57 & 0.49 & 1.70 & 0.99 \\
\hline
\end{tabular}

with a fair degree of accuracy. The coefficient of determination $\left(R^{2}\right)$ is significantly higher using Equation (4) than Equation (2). The Fickian diffusion model [Equation (2)] fails in the case of datasets that have a sharp knee. Since $\phi$ can also be interpreted as the fraction of equilibrium amount of sorption in the unrelaxed polymer in the fully relaxed polymer, ${ }^{[15]}$ the higher $\phi$ values of 50C/50 PBTA are reflection of low crystallinity of the continuous phase. For blends of chitosan and polyester, the $\psi$ values are similar $(0.32-0.75)$, indicating that the characteristic time of relaxation or the exchange between the two different modes is approximately 1.3-3.0 times of that of the diffusion time. For composites containing HA, the ratio ranges from 0.24 to 10.63 .

The diffusivity of composites containing chitosan and HA is higher than those without HA even though the equilibrium water uptake is lower. The results imply that the penetrant diffuses and reaches equilibrium faster as the mass of the polymer that adsorbs the solvent decreases (HA has very low water absorption). The interfacial region between the HA and the matrix benefits the transport of the penetrant (diffusant); in consequence, the water diffuses mainly through the HA-matrix interface, reaching the saturation faster than in the blends.

Using these values for diffusion coefficient, the water uptake profile was simulated using the first two terms and compared to the experimentally measured data. The simulated values are close to the experimentally measured ones for the entire duration (Figure 5). Equation (2) is derived under the assumption that the sample is homogeneous. The overall diffusion of water will depend on the degree of crystal-

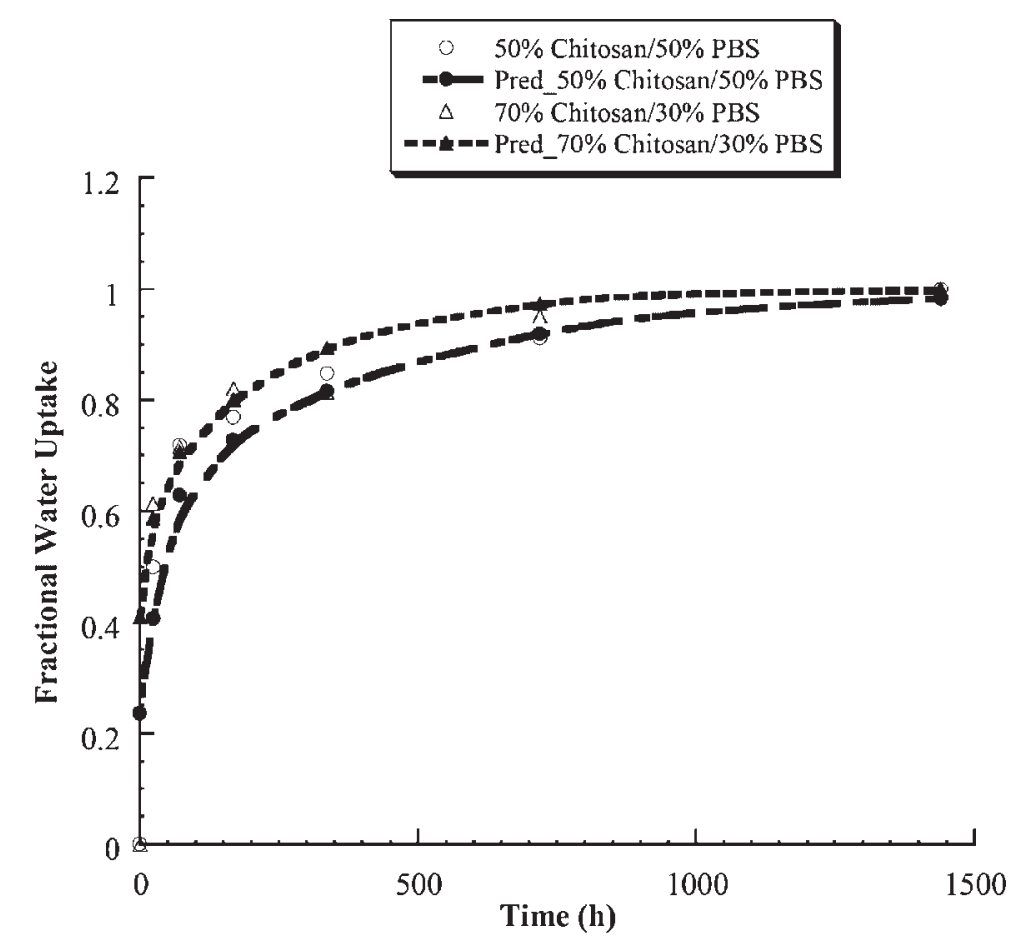

Figure 5. Comparison of predicted versus experimentally determined fractional water uptake for two different blend compositions. 
linity and the morphology of the blends and is positiondependent. From a morphological point of view, water diffusion is different in the continuous phase (polyester) versus the dispersed phase (chitosan). Hence, when the solvent enters the system, there is a non-homogeneous distribution of the diffusing solvent inside the polymeric matrix. One of the phases allows the penetrant to diffuse freely while the other immobilizes the penetrant, limiting its movement. The penetrant can become immobilized by reacting with functional groups on the polymers or getting lodged in holes and cavities. Perhaps a more appropriate model would have been the "dual-mode" model, ${ }^{[21]}$ which assumes that the transfer between the two modes can be described by a first-order reversible reaction. This leads to different relaxation times of polymer chains and, hence, anomalous effects in polymer-solvent diffusion. Three dimensionless numbers $\left(\theta=D t / L^{2}, \phi, \psi\right)$ are used to account for the water uptake in these materials. The relative magnitude of the rates of diffusion and relaxation processes is a major factor in determining the deviations from Fickian sorptions. ${ }^{[22]}$

Visual observations of samples after removal indicate some degree of swelling, particularly in blends containing higher amounts of chitosan (50\% and above). The diffusion coefficient increases with the concentration of chitosan due to the increased free volume caused by the swelling effect of the penetrating solvent. Solvent adsorbed can be lodged in holes and cavities, thus hindering the diffusion process. In addition, the penetrating solvent (water) is reversibly bound to the amine and hydroxyl groups in the chitosan domains dispersed within the polyester matrix, leaving only the unbound water to contribute to the diffusion. The largest deviation from Fick's model and the experimental data is obtained for chitosan and PLA. Of the polyester used in this study, PLA is the only polymer that has a glass transition temperature above the test temperature. The slow relaxation of the polymer chains due to the sorption of the solvent leads to deviation from the Fickian process. ${ }^{[23-26]}$

\section{Contact Angle}

There are no direct methods to measure surface energy or surface tension of solids. However, a number of indirect empirical and semi-empirical methods have been developed based on contact angle measurements. ${ }^{[27,28]}$ Kaelble's equation is very often used to determine surface tension of the blends because of its simplicity. Moreover, Kaelble's equation allows the determination of the polar components of the surface tension by measuring the contact angles of two liquids of known surface tension on the polymer surface:

$$
\begin{aligned}
& \gamma_{\mathrm{lv} 1}\left(1+\cos \theta_{1}\right) \\
& =2 \sqrt{\left(\gamma_{\mathrm{lv} 1}^{\mathrm{d}}+\gamma_{\mathrm{sv}}^{\mathrm{d}}\right)}+2 \sqrt{\left(\gamma_{\mathrm{lv} 1}^{\mathrm{p}}+\gamma_{\mathrm{sv}}^{\mathrm{p}}\right)} \\
& \gamma_{\mathrm{lv} 2}\left(1+\cos \theta_{2}\right) \\
& =2 \sqrt{\left(\gamma_{\mathrm{lv} 2}^{\mathrm{d}}+\gamma_{\mathrm{sv}}^{\mathrm{d}}\right)}+2 \sqrt{\left(\gamma_{\mathrm{lv} 2}^{\mathrm{p}}+\gamma_{\mathrm{sv}}^{\mathrm{p}}\right)}
\end{aligned}
$$

where the subscripts 1 and 2 represent the liquids glycerol and methyleneiodine, respectively. The polar and dispersion components of the surface tension of the blends are shown in Table 2 and were obtained after solving Equation

\begin{tabular}{|c|c|c|c|c|c|}
\hline \multirow[t]{2}{*}{ Material } & $\theta_{1}$ & $\theta_{2}$ & $\gamma^{\mathrm{p}}$ & $\gamma^{\mathrm{d}}$ & $\gamma$ \\
\hline & deg. & deg. & $\mathbf{m N} \cdot \mathbf{m}^{-2}$ & $\mathbf{m} \mathbf{N} \cdot \mathbf{m}^{-2}$ & $\mathbf{m} \mathbf{N} \cdot \mathbf{m}^{-2}$ \\
\hline PLA & 70.47 & 48.76 & 5.14 & 34.97 & 40.11 \\
\hline Chitosan (Chts) & 68.67 & 51.18 & 6.22 & 33.60 & 39.82 \\
\hline PBTA & 63.61 & 26.34 & 4.84 & 45.66 & 50.50 \\
\hline Chts/PBTA (50:50) & 73.60 & 48.64 & 4.04 & 35.03 & 39.07 \\
\hline PCL & 66.53 & 33.09 & 4.51 & 42.89 & 47.40 \\
\hline Chts/PCL (50:50) & 69.92 & 41.06 & 4.29 & 39.07 & 43.36 \\
\hline PBS & 67.23 & 45.53 & 5.85 & 36.73 & 42.58 \\
\hline Chts/PBS (70:30) & 69.95 & 37.33 & 3.97 & 40.93 & 44.90 \\
\hline Chts/PBS (50:50) & 61.30 & 40.02 & 7.32 & 39.60 & 46.92 \\
\hline Chts/PBS (25:75) & 57.24 & 34.86 & 8.20 & 42.09 & 50.29 \\
\hline
\end{tabular}
(5) and (6). The increase in the polar component is an

Table 2. Contact angle values ( $\theta_{1}$ with glycerol, $\theta_{2}$ with methyleneiodine), and surface tension components for chitosan and its blends with synthetic polyesters. 
indication of an increase in the polar groups existing on the surface.

PLA was used as a standard since it is a widely used biomaterial. Chitosan has a surface tension value similar to PLA (Table 2). Blending of chitosan with synthetic polyesters resulted in an increase in the surface tension over that of pure chitosan except in the case of chitosan/ PBTA where the surface tension was mostly unaffected. This was expected since those blends have been showing skin-core morphology with the skin being rich in polyester. ${ }^{[10]}$ The contact angle's values however were different from the ones measured for the pure synthetic polyesters. For PBTA and its blend with chitosan, which is mostly amorphous, the most significant difference was observed (surface tension of 50.50 and $39.07 \mathrm{mN} \cdot \mathrm{m}^{-2}$ respectively were calculated). A possible explanation for those observations could be an interaction such as hydrogen bonding between both the components that tie up the polar groups.

When the total surface free energies of the blends were separated into dispersive and polar components, the polar components exhibited a value close to that measured for pure synthetic polyesters. The magnitude of the dispersive components increased. The values obtained for the respective blends were between those of chitosan and those of polyesters, as was expected.

The influence of the chitosan/synthetic polyester ratios on the changes of surface hydrophilicity were studied for chitosan and PBS blends. Three different compounds with varying amounts of chitosan (Table 2) were studied. A decrease in the glycerol contact angle values was observed with the increase in synthetic component percentage. As a result, the highest surface tension of $50.29 \mathrm{mN} \cdot \mathrm{m}^{-2}$ was obtained for the lowest amount of chitosan in the blend chitosan/PBS (25:75). However, a different behavior for these blends was observed compared to the other two blends containing higher percentages of chitosan. Large changes in the polar component of the surface tension values from 3.97 to $8.20 \mathrm{mN} \cdot \mathrm{m}^{-2}$ were observed. There are two possible reasons for this observation. The first is related to the shorter aliphatic chain of PBS, and the second is the possible interaction/chemical bond between PBS and chitosan. In both cases, a reduced flexibility of the chain results in a more ordered and oriented surface structure. Moreover, it should be noted that the wettability could not always be directly correlated to the surface composition. ${ }^{[29]}$ When subjected to a change in environmental conditions, such as temperature or incubation medium, the surface composition can be altered by movements of certain components or groups to the surface. ${ }^{[30]}$ Surface composition can vary during the measurements due to the possible interactions between the two phases; glycerol, which has hydroxyl groups, could form hydrogen bonds with the hydroxyl groups from the material and in this way orient them from the bulk to the surface. These interactions can depend on both the $-\mathrm{OH}$ end groups concentration and the mobility of the block to which the $\mathrm{OH}$ belongs. On the other hand, diiodomethane and air do not have this ability. Hence, there is no interaction, which moves these groups to the material surface, thus affecting wettability.

\section{Degradation}

Pure polyesters show a very low percentage of water uptake. During the $60 \mathrm{~d}$ of immersion in the isotonic saline solution, the maximum water uptake was less than 1.5\% for all of them and the weight loss of the pure polyesters was almost insignificant - at less than $0.5 \%$. These results would indicate that there was minimal degradation. However, a plot of strain versus time curve for PBS immersed for different time periods in isotonic saline solution indicates that the degradation does occur (Figure 6). While the ultimate tensile strength (UTS) and the modulus (measured as the slope of the stress versus strain curve) remained invariant with time, a sharp decrease in the elongation to break was observed. The strain at break, decreased by over an order of magnitude after $60 \mathrm{~d}$ in an isotonic saline solution. Other polyesters showed a decrease in both strain and stress at break, indicating that there is a loss of mechanical properties without any appreciable weight loss. Of the other

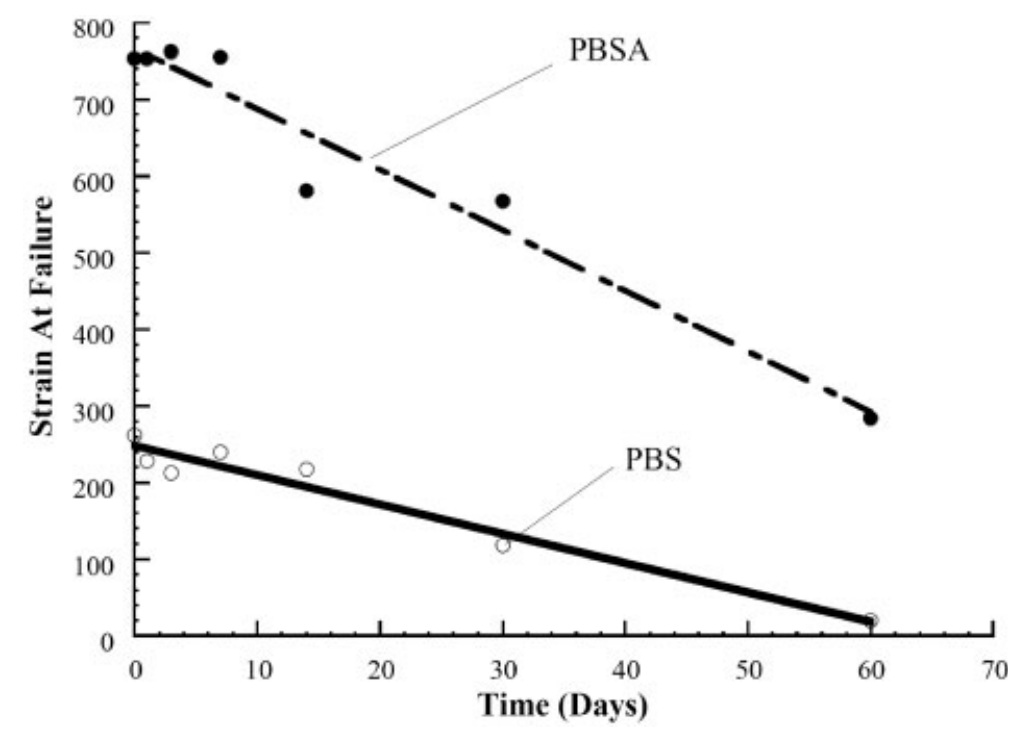

Figure 6. Strain at failure versus time of poly(butylenes succinate) and poly(butylene succinate adipate) as a function of immersion time in isotonic saline solution. 
polyesters, PLA had the largest decrease in tensile strength (15\%) and modulus (25\%) while PCL had the least. The rate of hydrolytic degradation for aliphatic polyesters depends primarily on the kinetics of the cleavage of the ester bonds. Furthermore, the degradation is much faster in amorphous domains than in crystalline domains, as water penetration is easier within a disordered network of polymer chains. The difference in the degradation rate between PLA and PCL is probably related to the crystallinity of the two polyesters. Hence, the low rate of degradation observed for PCL is expected since it has the highest crystallinity, which makes it resistant to hydrolytic degradation. An ester bond within a crystalline domain is much more resistant than the same bond within an amorphous domain. Initial degradation also causes an increase in the number of carboxylic ends, which autocatalyzes the ester hydrolysis. The changes in strain at break are a result of chain scission. Using a similar reasoning, degradation is faster in amorphous domains above the glass transition temperature than below the glass transition temperature because of the enhanced mobility of chain segments. The decrease in weight was insignificant because the degradation occurred without any leaching.

The change in the UTS as a function of immersion time for various blend compositions is shown in Figure 7. The UTS was normalized with respect to the initial tensile strength. For blends with the same chitosan content
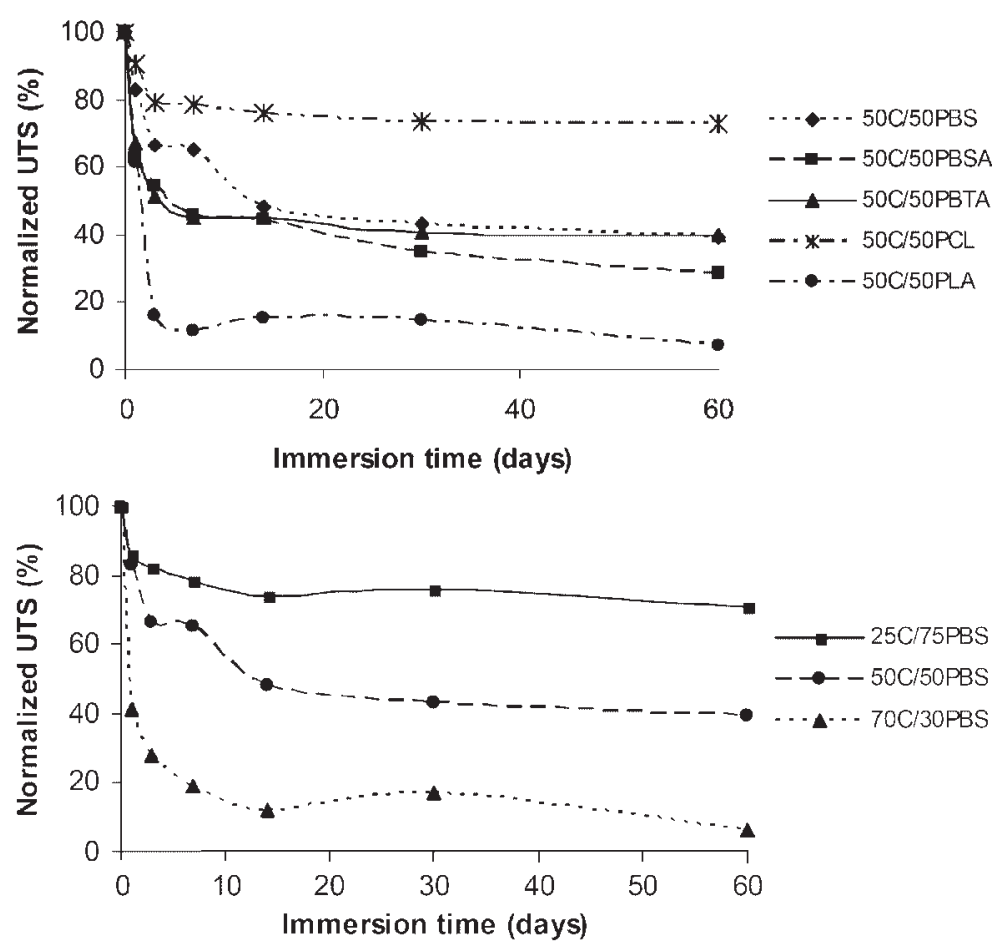

Figure 7. Degradation of mechanical properties of polyester/chitosan blends as a function of immersion time in isotonic saline solution. (a) Different polyester blends containing 50\% chitosan, (b) chitosan/PBS blends with different chitosan amounts.
[Figure 7(a)], the least degradation in terms of mechanical properties occurred for chitosan/PCL blends, while the most severe degradation occurred for chitosan/PLA blends. This is related to the fact that PCL is more crystalline and consequently less affected by hydrolytic degradation. PLA, on the other hand, is well known to be susceptible to hydrolytic degradation. ${ }^{[31,32]}$ The water uptake of chitosan/PCL blends is significantly lower than that of chitosan/PLA, and this leads to increased hydrolytic degradation of the latter. Blends containing PBS, PBSA, and PBTA show a similar degradation rate, with PBTA having a slightly higher initial rate.

As the chitosan content increased [Figure $7(b)$ ], the rate of decay of tensile strength increased. Blends containing 25\% chitosan with PBS had a degradation rate similar to that of blends containing 50\% chitosan with PCL, while blends containing $70 \%$ chitosan with PBS had a degradation rate similar to that of $50 \%$ chitosan with PLA (Figure 7). The same holds true for the modulus. This was a result of increased hydrolytic degradation due to increased water absorption. The deformation at break increased after degradation stages due to the presence of residual moisture that worked as a plasticizer. This residual water also contributes to the loss of stiffness. The loss in properties is primarily the effect of chitosan, which degrades and leaches out into the solution.

The change in mechanical properties during degradation as a function of HA content is given in Figure 8. For composites containing 30\% HA by weight, those blended with chitosan/PBTA showed a lower percentage loss than those blended with chitosan/PBS. There appears to be a little difference in the final value of the normalized tensile strength as a function of HA content. It should be noted that even though the normalized tensile strength is higher in some cases for composites containing $\mathrm{HA}$, the actual tensile strength for composites containing $\mathrm{HA}$ is always lower than those without HA.

The kinetics of degradation of the system was investigated by evaluating a plot of property loss with time. The rate of change of a component, $P$ is given by

$$
-\frac{\mathrm{d}[P]}{\mathrm{d} t}=k[P]^{n}
$$

Hence, a linear semi-log plot of $P$ versus time can be considered to follow a first-order kinetics. This appears to be the case of most of the data (with the exception of PCL and blends containing PCL). There appears to be some scatter in the data which do not lend to the estimation of the time constant $(k)$ with any reasonable degree of confidence. The fit for 


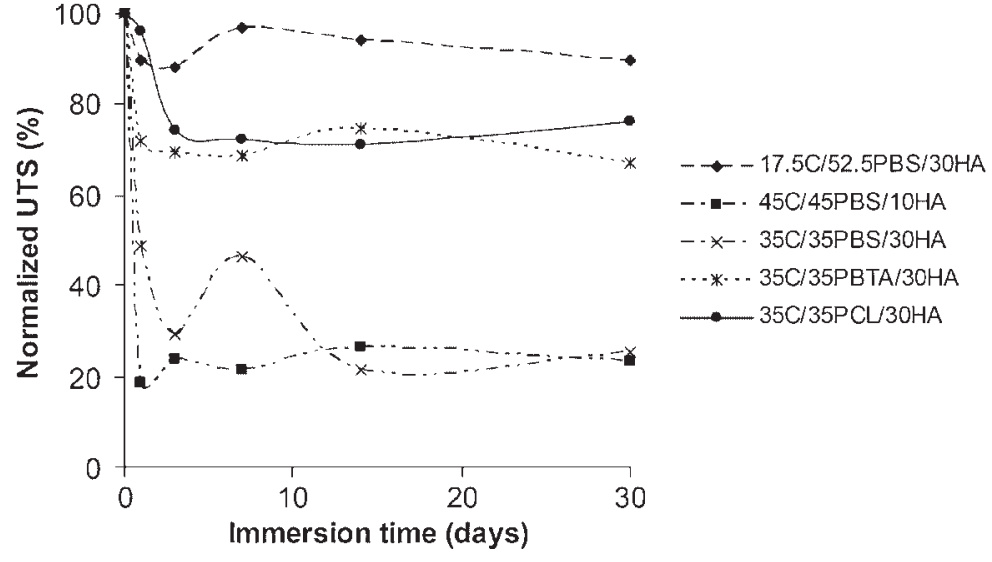

Figure 8. Degradation of mechanical properties of polyester/chitosan/HA blends as a function of immersion time in isotonic saline solution.

blends containing HA is poor, primarily because of the scatter in the data (Figure 8).

In terms of weight loss, the degradation behavior of all the chitosan/polyester blends and composites is typical of other natural-based biodegradable polymers. ${ }^{[33-37]}$ For all the blends and composites, there are two different degradation stages (Figure 7 and 8). The first degradation stage, between 0 and $7 \mathrm{~d}$, is characterized by a very fast water uptake and weight loss. In this stage, the mechanism responsible for this sharp increase in weight loss is a physical phenomenon. The higher amount of water uptake is responsible for the fast leaching of low molecular weight polymeric chains resulting from the thermomechanical degradation during processing. At the same time, the material swells and the water penetrates into the interface between the hydrophobic matrix and the hydrophilic disperse phase, releasing chitosan to the solution (and in some cases also HA). The chitosan release into the solution was responsible for the $\mathrm{pH}$ increase during the degradation periods (data not shown). This was independently verified by immersing $3.5 \mathrm{~g}$ of chitosan in $100 \mathrm{ml}$ of distilled water $(\mathrm{pH}=5.7)$ or buffer solution $(\mathrm{pH}=7.7)$. After $2 \mathrm{~h}$, the $\mathrm{pH}$ of both solutions increased significantly (10.0 for distilled water and 9.4 for the buffer). The chitosan release into the solution and consequent $\mathrm{pH}$ increase may influence the kinetics of degradation of polyesters. Wu et al. ${ }^{[38]}$ have shown that PLGA scaffolds coated with chitosan absorbed more water but had a slower degradation ratio than uncoated scaffolds. The release of chitosan material causes an increase in the $\mathrm{pH}$. This effect can neutralize the acidity associated with the degradation of PLGA. Thus, the degradation of chitosan can hinder the PLGA autocatalytic degradation and therefore retards its degradation kinetics.

The second degradation stage, between 15 and $60 \mathrm{~d}$, corresponds to stabilization in water uptake and weight loss. The principal process involved in the weight loss during this period is chemical degradation (hydrolysis). The weight loss is less pronounced because low molecular weight polymeric chains resulting from the thermal degradation and the chitosan particles were released during the first stage. In this stage, the weight loss slows down because only low molecular weight fractions resulting from the hydrolysis of the polyesters are released and the $\mathrm{pH}$ decreases. The polyester degradation proceeds by chain scission resulting from hydrolysis of the ester links until the molecular weight has decreased to the point where fragments are small enough to diffuse from the polymer matrix into the solution. Both these stages are characterized by a pronounced decrease in the UTS and modulus for all the blends. The strain at break for blends increased in all cases. Although the moisture present in the samples was stabilized before testing, some residual moisture was present. The residual moisture probably worked as a plasticizer.

The composites containing HA experienced higher weight loss. The weight loss increases as the HA content increases. This is related to the preferential attack at the polymeric-ceramic interface resulting in leaching of HA to the solution. Similar results were reported for other biodegradable polymers reinforced with HA. ${ }^{[34,35]}$ During the extrusion processing of blends containing HA, 5\% glycerol was added to enhance the plasticization process. The glycerol was released in the first degradation stage, contributing to the higher weight loss of chitosan-based blends containing HA. Furthermore, morphological studies of composites indicate poor adhesion between the HA and the matrix. ${ }^{[1]}$ Hence, during the sharp initial uptake of water due to the preferential location of chitosan in the outer circumference of the core, the solvent molecules penetrate the cavities between the HA and the matrix. This would lead to loosening of the HA from the matrix leading to increase in leaching and weight loss.

Since the addition of chitosan to the blends, results in higher water uptake (Figure 1), it also leads to higher weight loss. The blends with $70 \%$ chitosan have the highest weight loss (except the ones with HA), and the blends with $25 \%$ chitosan have the lowest weight loss. The increase in weight loss with the increase in chitosan present in the blend can be due to two mechanisms: both polyesters and chitosan degrade by hydrolysis. Higher water uptake promotes the hydrolysis and consequently the weight loss increases. Higher water uptake also promotes the erosion and diffusion of the chitosan particles from the matrix into the solution. The $\mathrm{pH}$ increased as a result of the release of chitosan particles into the solution. In the first degradation stage, the $\mathrm{pH}$ was higher for the blend containing 70 chitosan/30 PBS because the amount of chitosan released to the solution was higher. The fact that chitosan amount affects the degradation 
behavior of the blend is an important result because by controlling the chitosan percentage it is possible to tailor the degradation rate of the blend. In terms of weight loss, the blends containing the same amount of chitosan but different polyesters had a similar behavior.

\section{Conclusion}

The water absorption and degradation characteristics of chitosan-based polyesters and HA composites were studied. Pure polyesters showed hydrophobic behavior with low water uptake and displayed a decrease in the mechanical properties without any appreciable weight loss. Among the various polyesters studied, PLA showed the largest decrease in mechanical properties while PCL showed the least.

Increased chitosan content increased the water uptake and diffusion coefficient of the blends. For blends with the same chitosan content, the lowest equilibrium water content was observed in blends containing PCL, and the highest water uptake was experienced by the blends containing PLA. The water absorption of all blends and composites deviated from the Fickian diffusion model and was modeled using a two-stage sorption. Composites containing HA had a lower water uptake than blends containing Chitosan but achieved equilibrium water uptake much faster than the blends.

Increased chitosan content significantly reduced the mechanical properties and increased the weight loss as a function of immersion time. Chitosan/PCL showed the lowest degradation among the different blends with the same chitosan amount, whereas chitosan/PLA showed the most severe property degradation (corresponding to the lowest and the highest water uptake, respectively). This observation is an indication that the degradation of properties with immersion time was directly related to the water uptake of the blends. The composites containing HA experienced larger weight loss. The weight loss also directly correlates with the HA content, as expected.

Acknowledgements: V. M. Correlo would like to acknowledge the Portuguese Foundation for Science and Technology (FCT) for providing a grant (SFRH/BD/22455/2005). This work was carried out under the scope of the EU Integrated Project Genostem. M. B. would like to thank FLAD (Fundação Luso-Americana para o Desenvolvimento) for generous support of sabbatical funds.

Received: October 18, 2006; Revised: December 22, 2006; Accepted: January 12, 2007; DOI: 10.1002/mabi.200600233

Keywords: chitosan; chitosan blends; degradation; melt based; natural origin; water uptake

[1] C. A. Scotchford, B. Sim, S. Downes, M. Braden, Polymer 1997, 38,3869

[2] N. Suarez, S. Brocchini, J. Kohn, Biomaterials 1998, 19, 2347.
[3] L. Lu, C. A. Garcia, A. G. Mikos, J. Biomed. Mater Res. 1999, 46, 236.

[4] M. J. Lydon, T. W. Minett, B. J. Tighe, Biomaterials 1985, 6, 396.

[5] H. Sashiwa, J. M. Thompson, S. K. Das, Y. Shigemasa, S. Tripathy, R. Roy, Biomacromolecules 2000, 1, 303.

[6] R. G. Chapman, E. Ostuni, M. N. Liang, G. Melulent, E. Kim, L. Yan, G. Pier, H. S. Warren, G. M. Whitesides, Langmuir 2001, 17, 1225.

[7] G. Khang, J.-H. Choee, J. M. Rhee, H. B. Lee, J. Appl. Polym. Sci. 2002, 85, 1253.

[8] P. C. Schamberger, J. A. Gardella, Colloids Surf. B 1994, 3, 203.

[9] T. Koyano, N. Minoura, M. Nagura, K. Kobayashi, J. Biomed. Mater. Res. 1998, 39, 468.

[10] V. M. Correlo, L. F. Boesel, M. Bhattacharya, J. F. Mano, N. M. Neves, R. L. Reis, Mater. Sci. Eng. A 2005, 403, 57.

[11] V. M. Correlo, L. F. Boesel, M. Bhattacharya, J. F. Mano, N. M. Neves, R. L. Reis, Macromol. Mater. Eng. 2005, 290, 1157.

[12] R. Mani, M. Bhattacharya, Eur. Polym. J. 2001, 37, 515.

[13] H. Wang, X. Sun, P. Seib, J. Appl. Polym. Sci. 2002, 84, 1257.

[14] C. Santos, R. L. Clarke, M. Braden, F. Guitian, K. W. M. Davy, Biomaterials 2002, 23, 1897.

[15] Y. M. Sun, Polymer 1996, 37, 3921.

[16] H. Han, C. C. Gryte, M. Ree, Polymer 1995, 36, 1663.

[17] P. Nogueira, C. Ramirez, A. Torres, M. J. Abad, J. Cano, J. Lopez, I. Lopez-Bueno, L. Barral, J. Appl. Polym. Sci. 2001, 80, 71.

[18] K. Asaoka, S. Hirano, Biomaterials 2003, 24, 975.

[19] I. Sideridou, D. S. Achilas, C. Spyroudi, M. Karabela, Biomaterials 2004, 25, 367.

[20] J. Crank, "Mathematics of Diffusion", Second edition, Oxford University Press. Great Calendron Street Oxford OX2 6DP 1975.

[21] A. R. Berens, H. B. Hopfenberg, Polymer 1978, 19, 489.

[22] S. Joshi, G. Astarita, Polymer 1979, 20, 455.

[23] J. S. Vrentas, J. L. Duda, J. Polym. Sci., Polym. Phys. Ed. 1977, 15, 441.

[24] D. W. McCall, D. C. Douglass, L. L. Blyer, Jr., G. E. Johnson, L. W. Jelinski, H. E. Blair, Macromolecules 1984, 17, 1644.

[25] J. L. Valles, J. W. Halley, J. Chem. Phys. 1990, 92, 694.

[26] J. S. Vrentas, C. M. Jarzebski, J. L. Duda, AIChE J. 1975, $21,894$.

[27] B. D. Ratner, "Surface properties of materials", in: Biomaterials Science: An introduction to Materials in Medicine, B. D. Ratner, A. Hoffman, F. Schoen, J. Lemon, Eds., Academic Press, New York 1996, Vol. 1 , p. 21.

[28] Ch.-M. Chan, "Contact angle measurement", in: Polymer Surface Modification and Characterization, Hanser Publishers, Munich 1994.

[29] M. Toselli, M. Messori, R. Bongiovanni, G. Malucelli, A. Priola, F. Pilati, C. Tonelli, Polymer 2001, 42, 1771.

[30] J. P. Santerre, R. S. Labow, J. Biomed. Mater. Res. 1997, 36, 223.

[31] D. H. R. Ramkumar, M. Bhattacharya, Polym. Eng. Sci. 1998, 38, 1426.

[32] K. Jamshidi, S. H. Hyon, Y. Ikada, Polymer 1988, 29, 2229.

[33] R. L. Reis, S. C. Mendes, A. M. Cunha, M. J. Bevis, Polym Int. 1997, 43, 347.

[34] D. Demirgöz, C. Elvira, J. F. Mano, A. M. Cunha, E. Piskin, R. L. Reis, Polym. Degrad. Stab. 2000, 70, 161.

[35] M. Alberta Araújo, C. M. Vaz, A. M. Cunha, M. Mota, Polym. Degrad. Stab. 2001, 73, 237.

[36] C. M. Vaz, A. M. Cunha, R. L. Reis, Mater. Res. Innovations 2001 $4,375$.

[37] M. C. Azevedo, M. B. Claase, D. W. Grijpma, R. L. Reis, J. Feijen, J. Mater. Sci.: Mater. Med. 2003, 14, 103.

[38] Y.-C. Wu, S.-Y. Shaw, H.-R. Lin, T.-M. Lee, C.-Y. Yang, Biomaterials 2006, 27, 896. 